\title{
Distant Pulse Measurement System for Real-Time Surveillance Applications
}

\author{
Jaromir Przybyło $^{1}$, Mirosław Jabłoński ${ }^{1}$, Eliasz Kańtoch ${ }^{1}$, Piotr Augustyniak ${ }^{1}$ \\ ${ }^{1}$ AGH University of Science and Technology, Kraków, Poland
}

\begin{abstract}
Touchless heart-rate monitoring is desirable due to hygienic and discreet operation. Several approaches were proposed so far. Our design makes a step towards camera-based cardiac surveillance system. We have developed, and tested a real-time video processing algorithm. Viola-Jones body part detection framework was used for object detection. Than a Region Of Interest was determined for computation of the mean value of the selected components. A sequence of consecutive raw samples $y(t)$ constitutes a buffer subjected to normalization which is followed by noise reduction and limiting the band by means of a temporal FIR and FFT transformation. The pulse rate was detected as the frequency corresponding to maximum energy band in spectral domain. MATLAB was used for designing and testing the system and generated $C++$ code was compiled to a 32-bit floating point processor. We recorded footage from 2 volunteers at resolution $800 \times 600$ and data rate up to $150 \mathrm{~Hz}$. Wearable pulse oximeter was used for measuring reference signal. The error of estimated pulse of subjects under controlled illumination and during their physical activities ranged from 3.1 to $18.8 \mathrm{bmp}$. All computations meet demands of 60fps real time surveillance system, making the pulse-dedicated intelligent camera a handy tool for monitoring humans, their health status and emotions.
\end{abstract}

\section{Introduction}

Touchless measurements of human heart rate was found very interesting since a decade and started with a work by Verkruysse et al. [1], who proposed contactless measurement of the plethysmographic signal in ambient light with a regular video camera. This modality was improved thanks to approaches proposed by McDuff [2], Couderc [3], Królak [4] and many others inventors of new video sequence processing methods. Currently it's accuracy was proven competitive to wearable contact measurements of HR.

Introduction of new contactless heart rate measurement method allowed for hidden distant data collection and opened new areas of potential application of the heart rate signal. Emotion and workload analyses are only first examples at hand. Those usage scenarios do not assume a subject sitting still in good lighting conditions, and respective approaches try to compensate for light variations or subject's motion (e.g. [5] and [6]). Recently our group also contributed to this progress with studies of using a configurable frame-rate camera [7]. This paper draws the attention to the camera speed and possible video compression as potential source of $\mathrm{HR}$ measurement inaccuracy.

In this paper we make a step towards intelligent camera-based cardiac surveillance system by developing, implementing and testing a reliable real-time video processing algorithm. It consists of (1) automatic body part detection algorithm utilizing Viola-Jones object detection framework, (2) calculating the mean value of pixels of the selected component inside ROI and filtering and (3) transformation of sequence to the spectral domain and detection of dominant energy. Also, test framework utilizing MATLAB automatic code generation capabilities has been used, which greatly speeded up development process of the proposed algorithm.

\section{Implementation details}

In order to test the proposed algorithm by running it under varying conditions and monitor its behavior, a MATLAB environment (version R2017a) has been used as automated test framework (fig. A1). Using recorded video and ground truth signals from wearable heart rate sensor (Polar WearLink+) we have tested the proposed algorithm on recorded video sequences.

Then, the algorithm has been prepared for code generation by defining input/output data types and their sizes. This process has been facilitated by capabilities of the MATLAB Coder which helped identifying and fixing potential errors (i.e. buffer overflow, fixed-point conversion, unsupported functions). To ensure that our algorithm is ready for code generation, a MEX function has been automatically generated that wraps the compiled code for execution back within MATLAB. This allows iterating and fixing errors until the algorithm is suitable for code generation.

Finally, a C++ source code has been automatically generated from our MATLAB algorithm and compiled asa dynamically linked library (DLL). This library has 
been integrated with a $\mathrm{C}++$ application which acquires frames from industrial camera in real-time. This approach allowed us for continuous improvements and tests of the proposed algorithm.

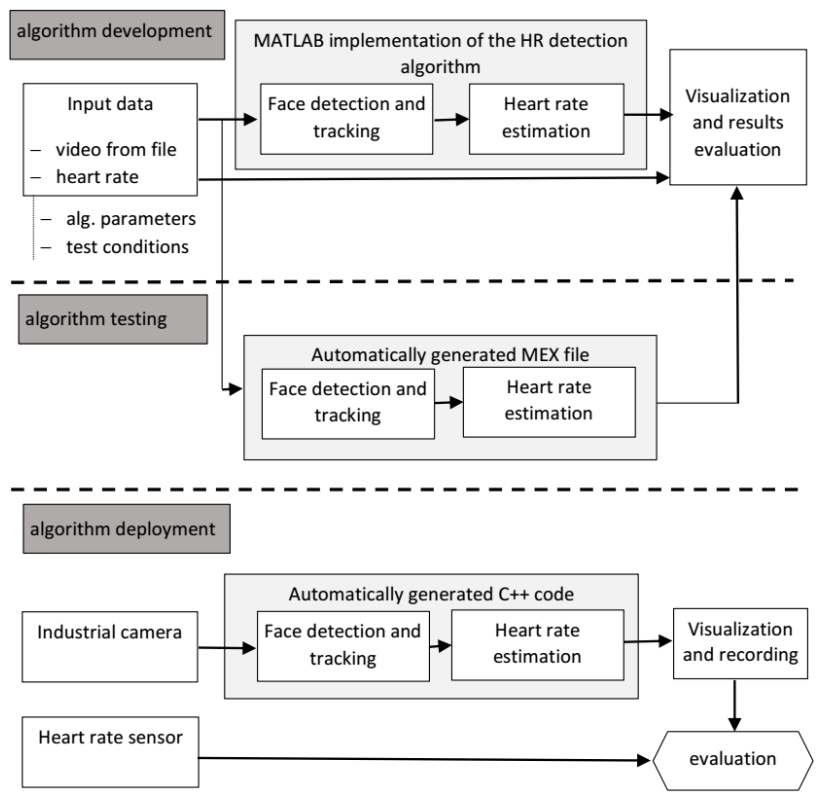

Fig. 1. Test framework.

\section{Algorithm description}

Detecting the heart rate from video sequences with a subject freely moving in the camera field of view requires detection and tracking of relevant body part (in our case the face). We used automatic body part detection algorithm employing Viola-Jones object detection framework [8]. On the first couple of frames, the face is detected. Then the Kanade-Lucas-Tomasi featuretracking algorithm [9] is used to track facial features. This allows reducing frame processing time. However, because tracked features can be lost in time, the face location has to be verified periodically and re-detected if necessary.

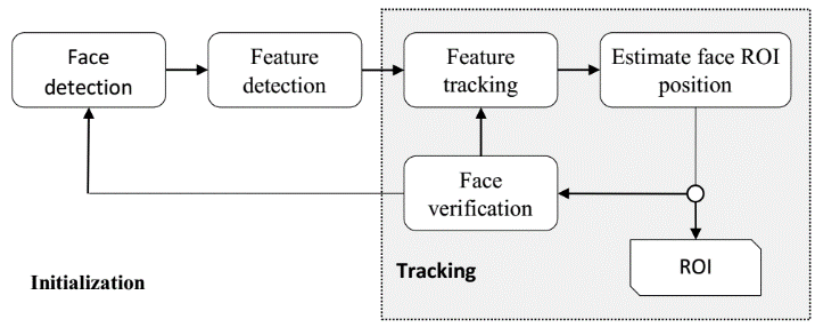

Fig. 2. Face detection and tracking algorithm.

On each frame of the video a Region of Interest (ROI) was computed based on the object detection and tracking results. It was then used to calculate the mean value of local color components inside ROI. From a sequence of consecutive frames, the raw signal $y(t)$ is formed in a buffer of length $N$. Then signal was normalized using formula (1):

$$
y^{\prime}(t)=\frac{y(t)-\mu}{\sigma}
$$

where $\mu$ and $\sigma$ are mean and standard deviation of $y(t)$ in the time window respectively. The recorded signal was then band-limited by a Bartlet-Hamming FIR filter (50$180 \mathrm{bpm}$ which is equivalent to $0.83-3.00 \mathrm{~Hz}$ ) and transformed to spectral domain via FFT. The pulse rate (named as $H R O$ in the paper) was detected as the frequency corresponding to maximum value of energy in the selected band (50-180 bpm). In order to minimize false detections, caused by head movements and other sources of image variation, estimated $H R$ was additionally post-processed. The second heart rate buffer of length $M$ has been used to store recent $H R 0$ values. Then a mean value of $H R$ buffer content has been computed and used as a new estimate of current heart rate (named as $H R 1$ in the paper). Additionally, when the difference between previous and current heart rate measurement is greater than a given threshold, this value is treated as outlier and weighted (2):

$$
\begin{aligned}
& H R 0_{i+1}= \\
& \left\{\begin{array}{cl}
H R 0_{i} & \text { if } a b s\left(H R 0_{i}-H R 1_{i-1}\right) \leq 3 f_{\text {res }} \\
w_{1} H R 0_{i}+\left(1-w_{1}\right) H R 1_{i-1} & \text { if abs }\left(H R 0_{i}-H R 1_{i-1}\right)>3 f_{\text {res }}
\end{array}\right\}
\end{aligned}
$$

where:

$$
\begin{array}{lll}
f_{\text {res }}- & \text { FFT frequency resolution, } \\
w_{1}- & \text { weighting factor }(0,1\rangle .
\end{array}
$$

\section{4. $\quad$ Results}

We recorded footage from 2 volunteers without particular positioning. The volunteers were asked to perform normal activities such as: talking with another person (facial actions), doing simple exercises e.g. lifting weights. The head movements and rotations resulting from above actions were within the accepted limits, that is camera's field of view. The length of video sequences was ranging from 1 to 3 minutes. Two different configurable machine vision cameras were used to measure the pulse under controlled illumination together with side low intensity daylight - Basler acA2000-165 $\mu \mathrm{mNIR}$ (monochrome camera., Conf. No. 2.) and Basler acA2000-165 $\mu$ c (color camera, Conf. No. 1). For color camera the green component was used to measure the heart rate. True value of pulse was controlled by Polar WearLink+ heart rate 
Table 1. The algorithm parameters used for experiments.

\begin{tabular}{|c|c|c|c|c|}
\hline Parameter & Conf. No. & a) & b) & c) \\
\hline bandpass filter order & \multirow{7}{*}{$\begin{array}{c}1 \\
\text { camera } \\
\text { color }\end{array}$} & 128 & 128 & 256 \\
\hline buffer length - seconds & & 10 & 20 & 30 \\
\hline length of FFT & & 1024 & 2048 & 4096 \\
\hline frame rate - fps & & & 100.0 & \\
\hline length of $H R$ window $M$ - seconds & & & 1.0 & \\
\hline image resolution & & & $640 \times 480$ & \\
\hline $\begin{array}{l}\text { Resulting frequency resolution in bpm } \\
\text { based on: FFT length / buffer length }(N)\end{array}$ & & $5.86 / 6.00(1024)$ & $2.93 / 3.00(2048)$ & $2.93 / 3.00(2048)$ \\
\hline bandpass filter order & \multirow{7}{*}{$\begin{array}{c}2 \\
\text { camera } \\
\text { mono }\end{array}$} & 256 & 512 & 512 \\
\hline buffer length - seconds & & 10 & 20 & 30 \\
\hline length of FFT & & 1024 & 204 & 4096 \\
\hline frame rate - fps & & & 150.0 & \\
\hline length of $H R$ window $M-$ seconds & & & 1.0 & \\
\hline image resolution & & & $640 \times 480$ & \\
\hline $\begin{array}{l}\text { Resulting frequency resolution in bpm } \\
\text { based on: FFT length / buffer length }(N)\end{array}$ & & $8.80 / 6.00(1024)$ & $4.40 / 3.00(2048)$ & $2.20 / 2.00(4096)$ \\
\hline
\end{tabular}

sensor. The distance of the subject to the camera was about $1.8 \mathrm{~m}$. As the ROI the forehead region has been used. The algorithm parameters used for experiments are summarized in table 1.

The buffer length was selected to match the FFT length as closely as possible. Then the zero-padding was applied (for example for 20 seconds and fps $=100, N=$ 2000 and padding was 48). The weight $w 1$ used was equal to 0.1 . Table 1 provides also the frequency resolution based on FFT length. It is worth to note that the resolution for shorter buffer length with zero padding is lower.

The reference $H R$ values were provided by WearLink+ heart rate sensor at irregular intervals usually every 1-3 seconds - which is result of the measurement method based on ECG. Because the heart rate values yielded from our algorithm were computed at camera sampling frequency, to compare the results with the reference we took running mean value of WearLink+ ground truth value computed in the time interval equal to FFT buffer length. Then the accuracy of estimated $H R$ was evaluated based on Root Mean Squared Error (RMSE) - table 2.

Increasing the FFT length provides a more accurate estimation of the heart rate. However, it also introduces greater latency in the measurements.

The performance of sequential $\mathrm{C}++$ implementation of the proposed algorithm was sufficient for a $60 \mathrm{fps}$ video sequence when using OpenCV image processing library and running on PC computer based on Intel Core i7-3610QM, 2.3GHz CPU-based.
Table 2. The accuracy of estimated $H R$.

\begin{tabular}{|c|c|c|c|c|}
\hline Subject & & & $\begin{array}{r}\text { HRO RMSE } \\
\mathrm{bpm}\end{array}$ & $\begin{array}{r}R{ }_{r}^{R M S E} \\
\mathrm{bpm}\end{array}$ \\
\hline \multirow{3}{*}{ male 1} & \multirow{3}{*}{1} & a) & 4.69 & 3.69 \\
\hline & & b) & 4.05 & 3.28 \\
\hline & & c) & 3.10 & 2.92 \\
\hline \multirow{3}{*}{ male 2} & \multirow{3}{*}{1} & a) & 12.49 & 11.37 \\
\hline & & b) & 10.30 & 9.93 \\
\hline & & c) & 9.97 & 9.91 \\
\hline \multirow{3}{*}{ male 1} & \multirow{3}{*}{2} & a) & 17.87 & 16.20 \\
\hline & & b) & 6.66 & 4.11 \\
\hline & & c) & 7.20 & 6.99 \\
\hline \multirow{3}{*}{$\begin{array}{l}\text { male } 1 \\
2^{\text {nd }} \text { test }\end{array}$} & \multirow{3}{*}{2} & a) & 18.85 & 17.70 \\
\hline & & b) & 14.23 & 13.51 \\
\hline & & c) & 15.52 & 15.42 \\
\hline
\end{tabular}

\section{Conclusions}

The video sequence based algorithm for distant heart rate measurements from a moving subject was developed and implemented in high level programming language (MATLAB) and integrated with $\mathrm{C}++$ application using MATLAB automated code generation capabilities. The resulting accuracy was increased thanks to using spectral domain analysis and estimated heart rate value postprocessing (averaging buffers). What is more important, the algorithm is now ready for implementation in intelligent camera embedded software. In the future, the computation can be accelerated by means of ubiquitous General Purpose GPUs able to process high resolution video streams [10] or custom hardware targeted for FPGA platforms. Such setups will allow for working with high speed or high resolution cameras allowing the 
subject for various movements in the field of view.

\section{Acknowledgements}

The scientific work is supported by the AGH University of Science and Technology in years 2017 as a research project No. 11.11.120.612.

\section{References}

[1] Verkruysse W., Svaasand L., Nelson S. Remote plethysmographic imaging using ambient light. Optics Express 2008; Vol. 16; No. 26: 21434-2144.

[2] McDuff D., Gontarek S., Picard R.W. Remote Detection of Photoplethysmographic Systolic and Diastolic Peaks Using a Digital Camera. IEEE Transactions on Biomedical Engineering 2014; Vol. 61; No 12: 2948-2955.

[3] Couderc JP., Kyal S., Mestha LK., Xu B., Peterson DR, Xia X., Hall B. Pulse Harmonic Strength of Facial Video Signal for the Detection of Atrial Fibrillation. Computing in Cardiology 2014; 41:661-664.

[4] Królak A. Influence of Skin Tone on Efficiency of VisionBased Heart Rate Estimation. In: Editor Augustyniak P., Maniewski R., Tadeusiewicz R. Recent Developments and Achievements in Biocybernetics and Biomedical Engineering, Proc. of. 20-th Polish Conference on Biocybernetics and Biomedical Engineering, Springer International Publishing AG, Series: Advances in Intelligent Systems and Computing, 2017:44-56.
[5] Jeanne V., Asselman M., Brinker B., Bulut M. Camerabased heart rate monitoring in highly dynamic light conditions. International Conference on Connected Vehicles and Expo (ICCVE) 2013:798-799.

[6] Li X., Chen J., Zhao G., Pietikainen M. Remote Heart Rate Measurement From Face Videos Under Realistic Situations. IEEE Conference on Computer Vision and Pattern Recognition (CVPR) 2014:4321-4328.

[7] Przybyło J., Kańtoch E., Jabłoński M., Augustyniak P. Distant measurement of plethysmographic signal in various lighting conditions using configurable frame-rate camera. Metrol. Meas. Syst. 2016; Vol. 23; No. 4: 579-592.

[8] Viola P., Jones MJ. Robust real-time face detection. International journal of computer vision 2004; 57(2):137154.

[9] Tomasi, C., Kanade T. Detection and tracking of point features. Technical Report CMU-CS-91-132, 493 Carnegie Mellon University, April 1991.

[10] Jabłoński M., Przybyło J. Evaluation of MoG video segmentation on GPU-based HPC system. Computing and Informatics, 2016, 35(5):1141-1159.

Address for correspondence.

Name. Jaromir Przybyło

Full postal address. 30 Mickiewicz Ave, 30-059 Krakow, Poland

E-mail address (optional).przybylo@agh.edu.pl 